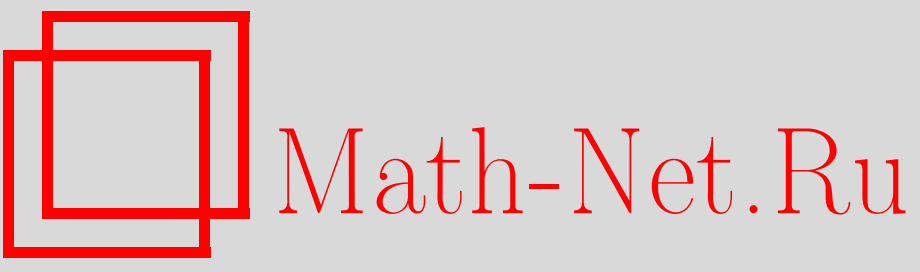

Н. М. Коробов, О конечных цепных дробях, УМН, 1997, том 52, выпуск 6, 167168

DOI: https://doi.org/10.4213/rm905

Использование Общероссийского математического портала Math-Net.Ru подразумевает, что вы прочитали и согласны с пользовательским соглашением http: //www . mathnet.ru/rus/agreement

Параметры загрузки:

IP: 34.239 .49 .27

26 апреля 2023 г., 18:16:17 


\title{
О КОНЕЧНЫХ ЦЕПНЫХ ДРОБЯХ
}

\author{
Н. М. Короьов
}

В настоящем сообщении предложен алгоритм, позволяющий, не прибегая к вычислению неполных частных, получить все несократимые правильные дроби $\frac{a}{m}$, для которых неполные частные разложения в цепную дробь не превосходят заданной величины $s \geqslant 2$. Как известно (см. [1]), такие дроби представляют интерес для теории приближенного интегрирования, так как числа $1, a$ будут оптимальными коэффицциентами по модулю $m$.

Обычно из двух разложений $\frac{a}{m}$ в цепную дробь выбирают то, в котором последнее неполное частное больше единицы. В отличие от этого, мы будем рассматривать разложения с последним неполным частным, равным единице. Рассмотрим группу дробей

$$
\frac{a(1)}{m(1)}, \ldots, \frac{a(\nu)}{m(\nu)} \quad(\nu \geqslant 1) .
$$

Строки, составленные из чисел

$$
a(1), \ldots, a(\nu) ; \quad m(1), \ldots, m(\nu) ; \quad m(1)+a(1), \ldots, m(\nu)+a(\nu)
$$

будем называть, соответственно, строкой числителей, строкой знаменателей и строкой сумми группьь (1).

Пусть $s \geqslant 2, \nu_{s} \geqslant \nu_{1}+\ldots, \nu_{s-1}$ и даны $s$ групп дробей

$$
\frac{a_{1}(1)}{m_{1}(1)}, \ldots, \frac{a_{1}\left(\nu_{1}\right)}{m_{1}\left(\nu_{1}\right)} ; \ldots ; \frac{a_{s}(1)}{m_{s}(1)}, \ldots, \frac{a_{s}\left(\nu_{s}\right)}{m_{s}\left(\nu_{s}\right)} .
$$

Группу дробей будем называть группой, порожденной группами (2), если для нее выполняются условия.

$1^{\circ}$. Строка числителей группы составлена из выписанных последовательно строк знаменателей групп (2), начиная с $s$-й и кончая первой из них:

$$
m_{s}(1), \ldots, m_{s}\left(\nu_{s}\right), m_{s-1}(1), \ldots, m_{s-1}\left(\nu_{s-1}\right), \ldots, m_{1}(1), \ldots, m_{1}\left(\nu_{1}\right) .
$$

$2^{\circ}$. Строка знаменателей группы состоит из двух частей. Первая ее часть совпадает со строкой сумм последней из групп (2), а вторая часть повторяет начальные числа первой части:

$$
\underbrace{\underbrace{m_{s}(1)+a_{s}(1), \ldots, m_{s}\left(\nu_{s}\right)+a_{s}\left(\nu_{s}\right)},}
$$

Так, например, при $s=2$ и $\nu_{2} \geqslant \nu_{1}$ группа дробей, порожденная группами

$$
\frac{a_{1}(1)}{m_{1}(1)}, \ldots, \frac{a_{1}\left(\nu_{1}\right)}{m_{1}\left(\nu_{1}\right)} ; \quad \frac{a_{2}(1)}{m_{2}(1)}, \ldots, \frac{a_{2}\left(\nu_{2}\right)}{m_{2}\left(\nu_{2}\right)}
$$

будет иметь вид

$$
\text { (3) } \frac{m_{2}(1)}{m_{2}(1)+a_{2}(1)}, \ldots, \frac{m_{2}\left(\nu_{2}\right)}{m_{2}\left(\nu_{2}\right)+a_{2}\left(\nu_{2}\right)}, \frac{m_{1}(1)}{m_{2}(1)+a_{2}(1)}, \ldots, \frac{m_{1}\left(\nu_{1}\right)}{m_{2}\left(\nu_{1}\right)+a_{2}\left(\nu_{1}\right)} \text {. }
$$

Дадим еще определение порядка последовательности дробей. При $s=2$ зададим две группы дробей:

$$
\frac{1}{2} ; \quad \frac{2}{3}, \frac{1}{3} \text {. }
$$

Каждую следующую группу будем выбирать так, чтобы она была порождена двумя предыдущими группами. В итоге получим последовательность

$$
\frac{1}{2} ; \frac{2}{3}, \frac{1}{3} ; \frac{3}{5}, \frac{3}{4}, \frac{2}{5} ; \frac{5}{8}, \frac{4}{7}, \frac{5}{7}, \frac{3}{8}, \frac{3}{7} ; \ldots .
$$

Действительно, в этой последовательности третья группа дробей

$$
\frac{3}{5}, \frac{3}{4}, \frac{2}{5},
$$


согласно порождающему алгоритму (3), получается из двух первых групп:

Аналогично, четвертая группа

$$
\frac{1}{2} ; \quad \frac{2}{3}, \frac{1}{3} \rightarrow \frac{3}{3+2}, \frac{3}{3+1}, \frac{2}{3+2} .
$$

получается из второй и третьей групп:

$$
\frac{5}{8}, \frac{4}{7}, \frac{5}{7}, \frac{3}{8}, \frac{3}{7}
$$

$$
\frac{2}{3}, \frac{1}{3} ; \frac{3}{5}, \frac{3}{4}, \frac{2}{5} \rightarrow \frac{5}{5+3}, \frac{4}{4+3}, \frac{5}{5+2}, \frac{3}{5+3}, \frac{3}{5+2},
$$

и т.д. Последовательность дробей (5), построенную с помощью начальных групп (4) и порождающего алгоритма (3), будем называть последовательностью второго порядка.

Пусть теперь $s \geqslant 3$. Выберем первые $s$ групп дробей так, чтобы они отличались от первых $s$ групп последовательности $(s-1)$-го порядка только тем, что конце $s$-й группы добавлена дробь $\frac{1}{s+1}$. Каждая следующая группа дробей выбирается так, чтобы она была порождена $s$ предыдущими группами. Полученную последовательность дробей будем называть последовательнос$m$ ью $s$-го порядка. Так, например, последовательность третьего порядка будет иметь вид

$$
\frac{1}{2} ; \frac{2}{3}, \frac{1}{3} ; \frac{3}{5}, \frac{3}{4}, \frac{2}{5}, \frac{1}{4} ; \frac{5}{8}, \frac{4}{7}, \frac{5}{7}, \frac{4}{5}, \frac{3}{8}, \frac{3}{7}, \frac{2}{7} ; \ldots
$$

Здесь первые три группы дробей отличаются от соответствующих групп последовательности второго порядка (5) тем, что в третьей группе добавлена дробь $\frac{1}{4}$. Каждая группа, начиная с четвертой, получается из трех предыдущих в соответствии с порождающим алгоритмом. В частности, четвертая группа

получается из трех первых групп:

$$
\frac{5}{8}, \frac{4}{7}, \frac{5}{7}, \frac{4}{5}, \frac{3}{8}, \frac{3}{7}, \frac{2}{7}
$$

$$
\frac{1}{2} ; \frac{2}{3}, \frac{1}{3} ; \frac{3}{5}, \frac{3}{4}, \frac{2}{5}, \frac{1}{4} \rightarrow \frac{5}{5+3}, \frac{4}{4+3}, \frac{5}{5+2}, \frac{4}{4+1}, \frac{3}{5+3}, \frac{3}{4+3}, \frac{2}{5+2},
$$
и т.д.

ТЕОРема. При каждом $s \geqslant 2$ последовательность $s$-го порядка содержит все те и только те правильные несократимые дроби, для которых неполные частные разложения в иепную дробь не превосходят $s$.

Доказательство теоремы проводится по индукции с использованием легко выявляемых особенностей разложений в цепную дробь чисел, когда дроби принадлежат одной и той же группе дробей последовательности $s$-го порядка.

ЗАмечАниЕ. Дроби, принадлежащие последовательности $s$-го порядка, удобно располагать в виде таблицы, составленной из строк числителей групп, образующих последователшность. Так, например, при $s=2$ получим следующую таблицу

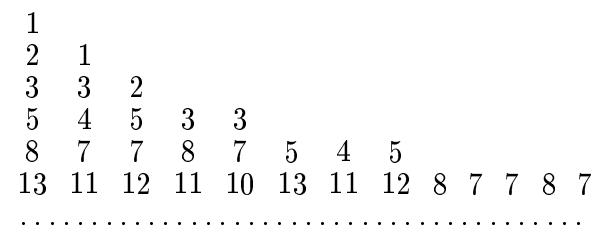

Отношения чисел из любой строки этой таблицы к стоящим под ними числам следующей строки образуют последовательность второго порядка (5) и, согласно утверждению теоремы, неполные частные их разложений в цепную дробь ограничены числом 2.

Считаю своим приятньм долгом отметить, что стимулом к появлению этого сообщения послужила статья [2].

\section{СПИСОК ЛИТЕРАТУРЫ}

[1] Коробов Н. М. Теоретико-числовые методы в приближенном анализе. М.: Физматгиз, 1963. [2] Мощевитин Н. Г. // Матем. заметки. 1996. Т. 2. С. 99-109.

Принято редколлегией 03.09.1997 